\title{
Proceedings of the 2018 next-generation Gamma Knife research meeting
}

\author{
Veronica L. Chiang, MD, ${ }^{1}$ Samuel T. Chao, MD, ${ }^{2}$ Constantin Tuleasca, MD, PhD, ${ }^{3}$ \\ Matthew C. Foote, MD, ${ }^{4}$ Cheng-chia Lee, MD, PhD, ${ }^{5}$ David Mathieu, MD, ${ }^{6}$ \\ Hany Soliman, MD, ${ }^{7}$ and Arjun Sahgal, MD ${ }^{7}$
}

'Department of Neurosurgery, Yale University, New Haven, Connecticut; '2Department of Radiation Oncology, Cleveland Clinic, Cleveland, Ohio; '3epartment of Clinical Neurosciences, Neurosurgery Service and Gamma Knife Center, Lausanne University Hospital (CHUV); Faculty of Biology and Medicine (FBM), University of Lausanne; and Signal Processing Laboratory (EPFL), Swiss Federal Institute of Technology, Lausanne, Switzerland; ${ }^{2}$ Department of Radiation Oncology, Princess Alexandra Hospital, University of Queensland, Brisbane, Queensland, Australia; ${ }^{5}$ Department of Neurosurgery, Neurological Institute, Taipei Veterans General Hospital; and School of Medicine, National Yan-Min University, Taipei, Taiwan; ${ }^{\circ D e p a r t m e n t ~ o f ~ N e u r o s u r g e r y, ~}$ Department of Surgery, Université de Sherbrooke, Quebec, Canada; and 'Department of Radiation Oncology, Sunnybrook Health Sciences Centre, University of Toronto, Ontario, Canada

In order to determine what areas of research are a clinical priority, a small group of young Gamma Knife investigators was invited to attend a workshop discussion at the 19th International Leksell Gamma Knife Society Meeting. Two areas of interest and the need for future radiosurgical research involving multiple institutions were identified by the young investigators working group: 1) the development of additional imaging sequences to guide the understanding, treatment, and outcome tracking of diseases such as tremor, radiation necrosis, and AVM; and 2) trials to clarify the role of hypofractionation versus single-fraction radiosurgery in the treatment of large lesions such as brain metastases, postoperative cavities, and meningiomas.

https://thejns.org/doi/abs/10.3171/2018.7.GKS181206

KEYWORDS Gamma Knife; novel imaging; tremor; radiation necrosis; hypofractionation; AVM; arteriovenous malformation; meningioma; brain metastasis; postoperative cavity consolidation; stereotactic radiosurgery

$\mathrm{S}$ INCE the first patient was treated with Gamma Knife stereotactic radiosurgery (GKSRS) in 1968, a significant body of research has been amassed to determine the clinical indications, optimum radiation dosing, and standardization of expected outcomes by using multicenter data. GKSRS is now in its 5th decade of use, and whereas the radiation source remains cobalt-60, the remainder of the machine has undergone significant changes, specifically to allow for automated treatment of multiple brain metastases and mask-based treatments facilitating the ease of delivery of hypofractionation dosing. However, with this change in its capability, many additional questions arise that need to be answered.

\section{Methods}

In order to determine what areas of research are a clinical priority, a small group of young Gamma Knife investigators was invited to attend a workshop discussion at the 19th International Leksell Gamma Knife Society Meeting. Members of this group were invited from 12 different countries and represented the subspecialties of neurosurgery (7 individuals), radiation oncology (9), physics (2), and radiology (1). Prior to the meeting, 3 neurosurgeons and 3 radiation oncologists who were considered early investigators on an academic track were chosen from this group by the leadership of the Leksell Gamma Knife Society. These 6 individuals were chosen because they had a track record of interest in research and proven academic productivity. All were asked to propose novel ideas that could lead to a potential research project to be completed using multicenter data accrual.

\section{Results and Discussion}

Proposals that were submitted fell under 2 main categories. 1) Need for more sophisticated advanced imaging techniques to better inform target definition for radiosur-

ABBREVIATIONS AVM = arteriovenous malformation; fMRI = functional MRI; GKSRS = Gamma Knife stereotactic radiosurgery; LINAC = linear accelerator; RTOG = Radiation Therapy Oncology Group; Vim = ventral intermediate thalamic nucleus; VimRS = Vim radiosurgery.

SUBMITTED May 1, 2018. ACCEPTED July 19, 2018.

INCLUDE WHEN CITING DOI: 10.3171/2018.7.GKS181206. 
gery and improve response assessment during imaging follow-up. 2) Need for more data to inform the role of hypofractionation for different disease processes.

\section{Imaging Techniques}

The first proposal addressed the need for better understanding of the radiobiology of radiosurgery of the ventral intermediate thalamic nucleus (Vim) for essential tremor, the most common movement disorder. ${ }^{21,22}$ The exact pathophysiological mechanisms of tremor are unknown, including its central versus peripheral origin. ${ }^{21} \mathrm{~A}$ common theory is that tremor arises from an abnormality within the "tremor axis," which includes the dentate cerebellar nucleus, the contralateral thalamus, and primary motor cortex. ${ }^{7,13}$ The existence of the axis is based on findings from noninvasive neuroimaging techniques, but also successful targeting of the Vim by the standard deep brain stimulation or stereotactic thermocoagulation, resulting in tremor arrest. ${ }^{5,13,15}$ Vim radiosurgery (VimRS), as an alternative to standard stereotactic procedures, has 3 major limitations: indirect targeting, no intraoperative confirmation of the Vim target by electrophysiology, and a delayed clinical effect of up to 1 year after the procedure. ${ }^{7,18,33,37}$

Tuleasca et al. have proposed using resting-state functional MRI (fMRI), ${ }^{30-32,34}$ a noninvasive neuroimaging technique that can be used to study spontaneous brain activity with minimal patient compliance, to study tremor pathophysiology and improve tremor arrest after VimRS, by using advanced computational techniques. ${ }^{6}$ Resting fMRI measures functional connectivity, which has been classically defined as the temporal correlations between spatially remote, statistically independent neurophysiological events distributed across varying neuronal groups and areas. ${ }^{14}$ These researchers' major findings include: 1) that pretherapeutic networks' interconnectivity can predict 1-year tremor arrest; and 2) discovery of a cerebellothalamo-visuo-motor network with a visually sensitive component, which is involved both in tremor generation and arrest after VimRS. This unexpected network has recently been confirmed by task-based fMRI studies and by findings from voxel-based morphometry. ${ }^{2,35}$ These findings have opened new gates for a better understanding of VimRS effects, but also raise the question of a new potential target for tremor (e.g., the visual association areas). The use of resting-state fMRI in observing this network over time might not only allow prediction of clinical response based only on pretherapeutic neuroimaging, ${ }^{29}$ but it might also facilitate a better understanding of the radiobiology of radiosurgical intervention and VimRS. ${ }^{30,32}$ This work by Tuleasca et al. would benefit both from validation in a larger cohort and from the expansion of its usefulness in Vim high-intensity focused ultrasound for tremor which, unlike radiosurgery, has an immediate clinical and radiological effect. ${ }^{9,10}$ A collaborative study would require uniformity of clinical assessment, including activities of daily living (from the questionnaire designed by Bain et al.), ${ }^{4}$ tremor score on the treated hand,,${ }^{12}$ and a quality of life questionnaire (QUEST).

The second proposal addressed the need for advanced imaging to better differentiate tumor recurrence from pseudoprogression and radiation necrosis after GKSRS for brain metastases. ${ }^{29}$ Given the lack of access in most facilities to amino acid-based PET imaging, different MRI sequences are used, including spectroscopy, diffusion, and perfusion, with variable sensitivity and specificity. Two commonly used sequences allowing perfusion imaging are dynamic contrast-enhanced imaging and dynamic susceptibility contrast imaging. Dynamic susceptibility contrast imaging is most commonly used and provides qualitative evaluation of relative cerebral blood volume by using simple color coding, whereas dynamic contrast-enhanced imaging measures quantitative cerebral blood volume and also allows evaluation of vascular permeability. ${ }^{36}$ Generally, an increase in perfusion would suggest tumor recurrence and a decrease would suggest radiation injury. It has been demonstrated that the use of both techniques leads to better sensitivity and specificity than the use of either one alone. ${ }^{17}$ Unfortunately, both imaging techniques require separate contrast injection, add additional time to the duration of the MRI for the patient, and require significant postprocessing. Because of this, most radiosurgeons still follow their patients with serial standard imaging to help with this diagnosis. Mathieu and imaging researchers in his institution have therefore developed a single hybrid imaging sequence from which both perfusion and permeability data can be extracted from each imaging pixel. This information can be fed automatically into a multiparametric analysis software system that then gives a pictorial and numerical probability of tumor recurrence versus nontumoral growth for each pixel analyzed. The multiparametric software is being developed using deep machine learning and will remove the need for onerous postprocessing by the radiologist. It is being developed as open-source software so that, if successful, it can be incorporated easily into multiple centers and validated based on clinical outcomes.

The third proposal addressed the need for better differentiation of arteriovenous malformation (AVM) structures for radiosurgery planning. Given the possible long survival of patients after successful GKSRS for AVM, late complications are a significant problem in approximately $30 \%$ of patients.$^{28}$ Late complications can include cyst formation, progressive vasogenic edema, and chronic expanding hematoma development. ${ }^{16,25,26}$ Preliminary data from Lee and colleagues (Lee CC et al., unpublished data) have shown that the risks of adverse radiation effects are reduced if radiation exposure of intervening normal brain is minimized, especially in diffuse forms of AVMs. These authors have therefore developed, using fuzzy c-means clustering techniques, a method of separating CSF, brain tissue, and AVM vasculature on high-resolution T2-weighted images, producing color-coded maps of the AVM showing these 3 components separately in order to help guide GKSRS planning. The issue is particularly important in light of the recent results from the ARUBA study (A Randomized Trial of Unruptured Brain Arteriovenous Malformations). ${ }^{24}$ Based on the results of Lee's report, patients with compact AVMs are still suitable candidates for treatment using GKSRS. The strict selection of patients could help to ensure that the administration of radiosurgery for compact, unruptured AVMs would provide a high likelihood of nidal obliteration with a low risk of 
adverse radiation effects. Furthermore, this group is looking to validate their techniques at other centers.

Discussion of these proposals by the larger group brought to light several areas of concern. Despite interest from many at the table, concern was raised about the challenges of cost for additional imaging and processing, and the ability to use some of the new sequences across different MR company platforms. For the thalamotomy project, the suggestion was made that imaging for identification of radiosurgery targets could be performed also in patients with tremor who are undergoing focused ultrasound treatment. Allying with this group could also help provide additional funding for this project. With regard to the tumor versus pseudoprogression project, additional MR sequences such as noncontrast chemical exchange saturation transfer (CEST) imaging or single-photon emission CT (SPECT) could also be added to the machine learning to see if they would provide additional value to the diagnostic process. ${ }^{23}$ The most significant concern about all the projects was the ability to subsequently import specialized testing results into GammaPlan without the use of intermediary third-party software.

\section{Hypofractionation}

Given the ability for Gamma Knife users to now perform mask-based hypofractionated treatments, radiation oncologists in the group were interested in discussing this technique for treatment of larger intracranial lesions.

The first proposal addressed meningiomas $>8-10 \mathrm{~cm}^{3}$ in volume. Treating these benign lesions using singlefraction 12- to 15-Gy GKSRS can result in an increased rate of neurological decline related to radiation toxicity. ${ }^{27}$ At the meeting, Foote therefore proposed doing a phase 3 randomized controlled trial to demonstrate superiority of administration of 25-27.5 Gy in 5 fractions as compared to a single fraction of $12-15$ Gy. The hypofractionation dose that was chosen as its biologically equivalent dose (BED) is equivalent to the single-fraction dose. ${ }^{8}$ Outcomes to be measured at 5 years post-GKSRS would be a composite end point including local tumor control rates and rates of neurological deterioration. The inclusion of 110 patients per arm should then be sufficient to demonstrate a $10 \%$ clinically significant difference in outcome between the 2 groups. Post hoc analysis of the data could then also examine the 2 outcomes stratified for additional factors, including meningioma location and postoperative versus empirical GKSRS.

The second proposal addressed larger brain metastases. Using the maximum tolerated dose determined from the Radiation Therapy Oncology Group (RTOG) 90-05 study, 15 or 18 Gy delivered to large brain metastases resulted in $50 \%$ local control at 1 year, ${ }^{28}$ much less than $85 \%$ local control seen with 24 Gy. RTOG 90-05 included patients with whole-brain radiation therapy, so the actual maximum tolerated dose actually may be higher in radiationnaïve patients. Although most GKSRS centers continue to use dosing guidelines from RTOG 90-05 and accept lower control, several centers have now started to propose alternative timing and dosing of SRS. Many radiosurgeons using linear accelerator (LINAC)-based therapy already use hypofractionation regimens for larger or multiple lesions, especially in the postresection setting. ${ }^{19,20}$ In contrast, radiosurgeons performing Gamma Knife therapy at the Cleveland Clinic use 2-stage radiosurgery ( 2 doses of 15 Gy, or 18 Gy then 12 Gy administered 1 month apart), and Asher et al. and Angelov et al. have described the successful use of lower-dose GKSRS as neoadjuvant radiosurgery prior to resection. ${ }^{1,3}$ As an alternative to Asher et al.'s protocol, the Cleveland Clinic has an in-house protocol with dose escalation for neoadjuvant radiosurgery. This latter technique allows for easier GKSRS planning because the margins are better defined, and preliminary data suggest that it also decreases the rate of leptomeningeal spread of the cancer at the time of surgery. At the meeting, Chao therefore proposed 2 possible phase 3 studies: 1) postoperative single-fraction RTOG 90-05 dosing GKSRS versus neoadjuvant SRS followed by resection versus hypofractionated GKSRS alone and 2) dose-staged SRS versus hypofractionated SRS (possible dosing to include 27 Gy in 3 fractions or 25-30 Gy in 5 fractions).

The third proposal addressed hypofractionation for postoperative cavities. As above, administration of GKSRS to postoperative cavities is challenged by the need to reduce dose based on increased volume, difficulty with defining the target accurately, difficulty differentiating tumor regrowth versus radiation necrosis after GKSRS, and variable definition of local recurrence. The proposed phase 3 study of approximately 140 patients would be a comparison of single-fraction to 5-fraction hypofractionated SRS (total dose ranging from 25 to $30 \mathrm{~Gy}$ ). The target definition, response assessment, and local failure evaluation would all be standardized and centrally reviewed. Outcomes to be measured would include local control rates and rates of radiation toxicity.

Discussion of these proposals by the larger group brought up several points of concern. The group in general agreed that there is a need to gather hypofractionated SRS data regarding whether surgery versus radiosurgery alone results in better local control for tumors $>3 \mathrm{~cm}$ in maximum diameter. The group also agreed on the need for data to help standardize hypofractionation regimens, because based on GKSRS there is a paucity of data to guide clinical practice, unlike for LINAC-based hypofractionated SRS. For brain metastases treated using GKSRS, a concern was raised regarding different radiobiological responses of different tumors to radiosurgery, especially when complicated by the use of targeted or checkpoint inhibitor systemic therapies. To take these many factors into account, studies including very large numbers of patients would be needed to see an effect. Based on 1999 salary and fringe benefit rates, Emanuel et al. reported a cost of slightly more than $\$ 6000$ (range $\$ 2098$ to $\$ 19,285$ ) per oncology patient to carry out a chemotherapeutic trial in 2003. ${ }^{11}$ Obtaining funding will therefore be one of the biggest challenges to gathering these data. Unlike pharmaceutical trials, in which the cost is offset by drug production if the trial yields positive results, there is no such payoff in a rigorously run randomized trial for singlefraction versus hypofractionated radiosurgery, especially given that one of the outcomes being tested is the risk of iatrogenic injury (radiation necrosis). Although a few centers remain interested in participating in a study without 
significant financial support, many find the cost prohibitive but agree that if funded, these data would very useful for guiding management decision-making in this current era of rapidly evolving technological capability.

Finally it was recognized that further discussions will be needed to move all of the above proposals forward. Although this meeting involved only Gamma Knife users, all of these topics are relevant to radiosurgery delivered across all platforms. The next venue for discussion will therefore probably be at the International Stereotactic Radiosurgery Society (ISRS) meeting in 2019 in Brazil. However, it has yet to be decided if discussions will be continued by the so-called next-generation group alone or, more likely, in conjunction with the newly renamed IRRF (International Radiosurgery Research Foundation-previously known as the North American Gamma Knife Consortium [NAGKC] and the International Gamma Knife Research Foundation [IGKRF]), through which discussions about project funding, study design, and data incorporation from the AANS/ASTRO (American Society for Radiation Oncology) radiosurgery registry across all SRS platforms could occur.

\section{Conclusions}

Two areas of interest and the need for future radiosurgical research were identified by the young investigators working group. First, there is a need to develop additional imaging sequences to better define disease process and treatment target and to improve patient follow-up. Second, trials are needed to define how to better treat larger lesions, including metastases and meningiomas, now that hypofractionation is a possibility with the Gamma Knife, in addition to the optimal treatment approach (single-fraction vs hypofractionated SRS) after surgery.

\section{Acknowledgments to the invited participants of the meeting}

Drs. Gerben R. Borst, Netherlands Cancer Institute; Jay Detsky, University of Toronto; Alex Dimitriadis, National Hospital for Neurology and Neurosurgery, London; Hirotaka Hasegawa, The University of Tokyo; Mustafa A. Hatiboglu, Bezmialem Vakif University, School of Medicine, Istanbul; Jin Wook Kim, Seoul National University Hospital; Kyung Hwan Kim, Samsung Medical Center, Korea; Rupesh Kotecha, Miami Cancer Institute, Baptist Health South Florida; Jing Li, MD Anderson Cancer Center, Houston, TX; Samir Patel, University of Alberta; Mark Ruschin, Odette Cancer Center, Toronto; Helena Sandstrom, Stockholm University; Daniel Trifiletti, Mayo Clinic, Rochester, MN; Manjul Tripathi, Postgraduate Institute of Medical Education and Research, India; Alvaro Villabona, National Hospital for Neurology and Neurosurgery, London; and Huai Che Yang, Taipei Veterans General Hospital.

\section{References}

1. Angelov L, Mohammadi AM, Bennett EE, Abbassy M, Elson P, Chao ST, et al: Impact of 2-staged stereotactic radiosurgery for treatment of brain metastases $\geq 2 \mathrm{~cm}$. J Neurosurg 22:1-17, 2017

2. Archer DB, Coombes SA, Chu WT, Chung JW, Burciu RG, Okun MS, et al: A widespread visually-sensitive functional network relates to symptoms in essential tremor. Brain 141:472-485, 2018

3. Asher AL, Burri SH, Wiggins WF, Kelly RP, Boltes MO, Mehrlich M, et al: A new treatment paradigm: neoadjuvant radiosurgery before surgical resection of brain metastases with analysis of local tumor recurrence. Int J Radiat Oncol Biol Phys 88:899-906, 2014

4. Bain PG, Findley LJ, Atchison P, Behari M, Vidailhet M, Gresty M, et al: Assessing tremor severity. J Neurol Neurosurg Psychiatry 56:868-873, 1993

5. Benabid AL, Pollak P, Gao D, Hoffmann D, Limousin P, Gay $E$, et al: Chronic electrical stimulation of the ventralis intermedius nucleus of the thalamus as a treatment of movement disorders. J Neurosurg 84:203-214, 1996

6. Biswal B, Yetkin FZ, Haughton VM, Hyde JS: Functional connectivity in the motor cortex of resting human brain using echo-planar MRI. Magn Reson Med 34:537-541, 1995

7. Buijink AW, van der Stouwe AM, Broersma M, Sharifi S, Groot PF, Speelman JD, et al: Motor network disruption in essential tremor: a functional and effective connectivity study. Brain 138:2934-2947, 2015

8. Conti A, Pontoriero A, Midili F, Iatì G, Siragusa C, Tomasello C, et al: CyberKnife multisession stereotactic radiosurgery and hypofractionated stereotactic radiotherapy for perioptic meningiomas: intermediate-term results and radiobiological considerations. Springerplus 4:37-44, 2015

9. Elias WJ, Huss D, Voss T, Loomba J, Khaled M, Zadicario E, et al: A pilot study of focused ultrasound thalamotomy for essential tremor. N Engl J Med 369:640-648, 2013

10. Elias WJ, Lipsman N, Ondo WG, Ghanouni P, Kim YG, Lee $\mathrm{W}$, et al: A randomized trial of focused ultrasound thalamotomy for essential tremor. N Engl J Med 375:730-739, 2016

11. Emanuel EJ, Schnipper LE, Kamin DY, Levinson J, Lichter AS: The costs of conducting clinical research. J Clin Oncol 21:4145-4150, 2003

12. Fahn S, Tolosa E, Marín C: Clinical rating scale for tremor, in Jankovik J, Tolosa E: Parkinson's Disease and Movement Disorders. Baltimore: Urban \& Schwarzenberg, 1988, pp 225-234

13. Fang W, Chen H, Wang H, Zhang H, Puneet M, Liu M, et al: Essential tremor is associated with disruption of functional connectivity in the ventral intermediate nucleus-motor cortex-cerebellum circuit. Hum Brain Mapp 37:165-178, 2016

14. Fox MD, Corbetta M, Snyder AZ, Vincent JL, Raichle ME: Spontaneous neuronal activity distinguishes human dorsal and ventral attention systems. Proc Natl Acad Sci U S A 103:10046-10051, 2006

15. Goldman MS, Ahlskog JE, Kelly PJ: The symptomatic and functional outcome of stereotactic thalamotomy for medically intractable essential tremor. J Neurosurg 76:924-928, 1992

16. Ilyas A, Chen CJ, Ding D, Mastorakos P, Taylor DG, Pomeraniec IJ, et al: Cyst formation after stereotactic radiosurgery for brain arteriovenous malformations: a systematic review. J Neurosurg 128:1354-1363, 2018

17. Koh MJ, Kim HS, Choi CG, Kim SJ: Which is the best advanced MR imaging protocol for predicting recurrent metastatic brain tumor following gamma-knife radiosurgery: focused on perfusion method. Neuroradiology 57:367-376, 2015

18. Kondziolka D, Ong JG, Lee JY, Moore RY, Flickinger JC, Lunsford LD: Gamma Knife thalamotomy for essential tremor. J Neurosurg 108:111-117, 2008

19. Lima LC, Sharim J, Levin-Epstein R, Tenn S, Teles AR, Kaprealian T, et al: Hypofractionated stereotactic radiosurgery and radiotherapy to large resection cavity of metastatic brain tumors. World Neurosurg 97:571-579, 2017

20. Lockney NA, Wang DG, Gutin PH, Brennan C, Tabar V, Bal- 
langrud A, et al: Clinical outcomes of patients with limited brain metastases treated with hypofractionated $(5 \times 6 \mathrm{~Gy})$ conformal radiotherapy. Radiother Oncol 123:203-208, 2017

21. Louis ED: Essential tremor. Lancet Neurol 4:100-110, 2005

22. Louis ED: Essential tremors: a family of neurodegenerative disorders? Arch Neurol 66:1202-1208, 2009

23. Mehrabian H, Desmond KL, Soliman H, Sahgal A, Stanisz GJ: Differentiation between radiation necrosis and tumor progression using chemical exchange saturation transfer. Clin Cancer Res 23:3667-3675, 2017

24. Mohr JP, Parides MK, Stapf C, Moquete E, Moy CS, Overbey JR, et al: Medical management with or without interventional therapy for unruptured brain arteriovenous malformations (ARUBA): a multicentre, non-blinded, randomised trial. Lancet 383:614-621, 2014

25. Parkhutik V, Lago A, Aparici F, Vazquez JF, Tembl JI, Guillen L, et al: Late clinical and radiological complications of stereotactical radiosurgery of arteriovenous malformations of the brain. Neuroradiology 55:405-412, 2013

26. Pollock BE, Gorman DA, Coffey RJ: Patient outcomes after arteriovenous malformation radiosurgical management: results based on a 5- to 14-year follow-up study. Neurosurgery 52:1291-1297, 2003

27. Pollock BE, Stafford SL, Link MJ, Garces YI, Foote RL: Single-fraction radiosurgery of benign cavernous sinus meningiomas. J Neurosurg 119:675-682, 2013

28. Shaw E, Scott C, Souhami L, Dinapoli R, Kline R, Loeffler J, et al: Single dose radiosurgical treatment of recurrent previously irradiated primary brain tumors and brain metastases: final report of RTOG protocol 90-05. Int J Radiat Oncol Biol Phys 47:291-298, 2000

29. Stockham AL, Tievsky AL, Koyfman SA, Reddy CA, Suh JH, Vogelbaum MA, et al: Conventional MRI does not reliably distinguish radiation necrosis from tumor recurrence after stereotactic radiosurgery. J Neurooncol 109:149-158, 2012

30. Tuleasca C, Najdenovska E, Régis J, Witjas T, Girard N, Champoudry J, et al: Clinical response to Vim's thalamic stereotactic radiosurgery for essential tremor is associated with distinctive functional connectivity patterns. Acta Neurochir (Wien) 160:611-624, 2018

31. Tuleasca C, Najdenovska E, Régis J, Witjas T, Girard N, Champoudry J, et al: Pretherapeutic functional neuroimaging predicts tremor arrest after thalamotomy. Acta Neurol Scand 137:500-508, 2018

32. Tuleasca C, Najdenovska E, Régis J, Witjas T, Girard N, Champoudry J, et al: Ventrolateral motor thalamus abnormal connectivity in essential tremor before and after thalamotomy: a resting-state functional magnetic resonance imaging study. World Neurosurg 113:e453-e464, 2018

33. Tuleasca C, Pralong E, Najdenovska E, Cuadra MB, Marques
JRF, Vingerhoets F, et al: Deep brain stimulation after previous gamma knife thalamotomy of the Vim for essential tremor is feasible! Clinical, electrophysiological and radiological findings. Acta Neurochir (Wien) 159:1371-1373, 2017

34. Tuleasca C, Régis J, Najdenovska E, Witjas T, Girard N, Champoudry J, et al: Pretherapeutic functional imaging allows prediction of head tremor arrest after thalamotomy for essential tremor: the role of altered interconnectivity between thalamolimbic and supplementary motor circuits. World Neurosurg 112:e479-e488, 2018

35. Tuleasca C, Witjas T, Van de Ville D, Najdenovska E, Verger A, Girard N, et al: Right Brodmann area 18 predicts tremor arrest after Vim radiosurgery: a voxel-based morphometry study. Acta Neurochir (Wien) 160:603-609, 2018

36. Welker K, Boxerman J, Kalnin A, Kaufmann T, Shiroishi M, Wintermark M: ASFNR recommendations for clinical performance of MR dynamic susceptibility contrast perfusion imaging of the brain. AJNR Am J Neuroradiol 36:E41E51,2015

37. Witjas T, Carron R, Krack P, Eusebio A, Vaugoyeau M, Hariz M, et al: A prospective single-blind study of Gamma Knife thalamotomy for tremor. Neurology 85:1562-1568, 2015

\section{Disclosures}

Dr. Sahgal has given educational seminars for Elekta AB; Accuray, Inc.; and Varian Medical Systems. He has a research grant from Elekta $\mathrm{AB}$, and has received travel accommodations and/or expenses from Elekta and Varian. Dr. Sahgal also belongs to the Elekta MR LINAC Research Consortium.

\section{Author Contributions}

Conception and design: Chiang, Sahgal. Acquisition of data: Chiang, Chao, Tuleasca, Foote, Lee, Mathieu, Soliman. Analysis and interpretation of data: Chiang. Drafting the article: Chiang. Critically revising the article: Chiang. Reviewed submitted version of manuscript: Chiang. Approved the final version of the manuscript on behalf of all authors: Chiang.

\section{Supplemental Information \\ Previous Presentation}

This material was presented and discussed at the 19th International Leksell Gamma Knife Society Meeting in Dubai, United Arab Emirates, on March 4, 2018.

\section{Correspondence}

Veronica Chiang: Yale University, New Haven, CT. veronica. chiang@yale.edu. 